\title{
Hypervigilance in College Students: Associations With Betrayal and Dissociation and Psychometric Properties in a Brief Hypervigilance Scale
}

\author{
Rosemary E. Bernstein and Brianna C. Delker \\ University of Oregon
}

\author{
Jeffrey A. Knight \\ Boston University School of Medicine
}

\author{
Jennifer J. Freyd \\ University of Oregon
}

\begin{abstract}
Betrayal trauma theory (Freyd, 1994, 1996) proposes that traumas high in social betrayal are expected to lead to psychological outcomes of dissociation, amnesia, and/or shame because these responses are adaptive to a survivor trying to preserve a necessary relationship in the face of mistreatment. Within the field of trauma studies more generally, there is substantial support for the proposition that traumas that cause intense fear should lead to posttraumatic anxiety and hypervigilance. Despite ample evidence for both theorized causal pathways, very few studies have tested associations between betrayal exposure, hypervigilance, and dissociation. The current study had 2 aims: first, as no self-report measure of hypervigilance had been developed for nonveteran populations, we sought to identify a subset of Hypervigilance Questionnaire (Knight, 1993) items that validly and reliably measure hypervigilance within college undergraduates ( $n=489 ; 62.6 \%$ female, $69.9 \%$ Caucasian) with and without elevated levels of posttraumatic stress. Second, we tested the associations among trauma history, hypervigilance, and dissociation. Psychometric analyses revealed 5 hypervigilance items we introduce as the Brief Hypervigilance Scale. Partial correlations revealed that each posttraumatic response was not related to a history of low betrayal trauma (i.e. non-interpersonal trauma) controlling for betrayal trauma (i.e. interpersonal trauma), but was related to betrayal trauma controlling for low betrayal trauma. These associations remained significant after controlling for the other posttraumatic response (i.e. hypervigilance or dissociation). Follow-up analyses revealed that hypervigilance was independently associated with adult, but not child high betrayal trauma, and the opposite was true for dissociation. Implications for theory, research, and clinical practice are discussed.
\end{abstract}

Keywords: betrayal trauma, dissociation, hypervigilance, measurement development

Hypervigilance-literally, the state of being "carefully watchful for possible danger or difficulties" (-vigilance) "to an excessive degree" (-hyper), and dissociation- "a lack of normal integration of thoughts, feelings, and experiences into the stream of consciousness and memory" (Bernstein \& Putnam, 1986 , p. 727) are both common psychological responses to trauma that are associated with a range of functional impairment, particularly disturbances in cognition, attention, and appraisal of threat (Briere, 2006; Cisler et al., 2011). Relatively little is known, however, about the association between dissociation and hypervigilance, and how the two might manifest within individuals. In some respects, they appear to be conceptual antonyms-with dissociation reflecting full or partial un-

This article was published Online First June 29, 2015.

Rosemary E. Bernstein and Brianna C. Delker, Department of Psychology, University of Oregon; Jeffrey A. Knight, Department of Psychiatry, Boston University School of Medicine; Jennifer J. Freyd, Department of Psychology, University of Oregon.

Correspondence concerning this article should be addressed to Rosemary E. Bernstein, Department of Psychology, 1227 University of Oregon, Eugene, OR 97403-1227. E-mail: reb@uoregon.edu awareness of traumatic information, and hypervigilance reflecting chronic access to traumatic information. However, if and when hypervigilance is motivated by implicit memories of trauma not integrated into explicit or autobiographical memory; or when it is generalized or displaced onto settings not directly relevant to the traumatic experience(s), dissociation and hypervigilance remain wholly compatible. In the present article, we review the relevant theoretical and empirical literature, introduce a new brief measure of hypervigilance, and describe an original exploratory study examining these relationships in an undergraduate sample.

\section{Fear and Betrayal}

Betrayal Trauma Theory (BTT; Freyd, 1994, 1996) is a theory of psychological response to trauma that proposes that an individual's response to trauma should depend on two independent features of the trauma: (a) the degree to which it involved social betrayal, and (b) the degree to which it induced terror or fear. More specifically, BTT argues that traumas high in betrayal should lead to dissociation, numbing, amnesia, and/or shame. Traumas that are extremely frightening, on the other hand, have long been thought to lead to hypervigilance, hyperarousal, and/or anxiety (e.g., Brett, 
1996). ${ }^{1}$ These hypotheses are deeply rooted in an evolutionary perspective, with each posttraumatic response serving an adaptive function: betrayed individuals should be motivated to remain "blind to betrayal" when awareness may elicit withdrawal or retaliatory behaviors that threaten a needed (or apparently needed) relationship, and terrorized individuals should be acutely attuned to signs of potential danger as a way to protect themselves against further traumatization.

Empirical support for these proposed links is plentiful. Indeed, a large and growing body of work has shown that betrayal-and not fear-is strongly associated with dissociation (see DePrince \& Freyd, 2007 for review). Betrayal has also been linked to shame, depression, inexplicable somatic symptoms (e.g., intermittent paralysis), and substance abuse-all of which are at least marginally related to the concept of dissociative unawareness (Freyd, 1996; Goldsmith, Freyd, \& DePrince, 2012; Hailey, 2014; Martin, Cromer, DePrince, \& Freyd, 2013). The link between terror and hypervigilance is also well elaborated within the traumatic stress field, which often places pathological fear at the core of posttraumatic stress (i.e., the "fear paradigm"; DePrince \& Freyd, 2002). In fact, many of the researchers who study PTSD (a hallmark symptom of which is hypervigilance) have suggested that it is a disorder of fear conditioning - that individuals with PTSD learn fear associates quickly and unlearn them slowly (Amstadter, Nugent, \& Koenen, 2009; Fanselow \& Ponnusamy, 2008; Milad et al., 2008; Peri, Ben-Shakhar, Orr, \& Shalev, 2000).

Surprisingly, despite the richness of these two literature bases, there has been very little research on the association between hypervigilance and dissociation following trauma. In one of the few exceptions, Kimble, Fleming, and Bennion (2013) tested whether dissociation negatively predicted hypervigilance in a sample of traumatized veterans with and without PTSD. The authors found that although dissociation and hypervigilance were associated in zero order correlations, when PTSD symptoms were accounted for, dissociation no longer predicted hypervigilance. The authors concluded that hypervigilance indicates the presence of PTSD and not the "absorption and depersonalization" associated with dissociation (Kimble et al., 2013). Because military training inculcates and reinforces vigilance, it is unclear how well these findings generalize beyond veteran populations (Kimble et al., 2013).

\section{Measuring Hypervigilance}

Although hypervigilance is a well-used clinical term associated with a range of anxiety and personality disorders (De Cort, Hermans, Spruyt, Griez, \& Schruers, 2008; Freeman, Garety, \& Phillips, 2000; Sposari \& Rapee, 2007) and with Posttraumatic Stress Disorder (PTSD) in particular (Jones \& Wessely, 2005; Kimble et al., 2013), less research on the construct has been published than one might expect. ${ }^{2}$ Within the small but rich body of extant literature, researchers have operationalized hypervigilance in a wide variety of ways. Most have measured it behaviorally, as distributions of visual attention captured with eye-tracking software (e.g., Huijding, Mayer, Koster, \& Muris, 2011; Wieser, Pauli, Weyers, Alpers, \& Mühlberger, 2009); RTs and accuracy scores on emotional Stroop (e.g., Arntz, Appels, \& Sieswerda, 2000; Peach, Jovev, Foster, \& Jackson, 2012) or attentional deployment tasks (e.g., Dalgleish, Moradi, Taghavi, Neshat-Doost, \& Yule,
2001); size estimation precision (Rhodewalt \& O'Keefe, 1986); or, in one study with children, as elevated picture-completion subtest scores relative to other subtests on a standardized intelligence test (Frankel, Boetsch, \& Harmon, 2000).

Although these studies have contributed greatly to the basic science of hypervigilance, these behavioral measures are not particularly accessible to researchers interested in indexing peoples' hypervigilant behaviors, nor are they able to capture hypervigilance as it manifests in the real world. A popular assessment of PTSD symptoms, the PTSD Checklist-Civilian Version (PCL-C; Blanchard, Jones-Alexander, Buckley, \& Forneris, 1996) is better suited to capture real-life behavior, but measures hypervigilance as a single-item component of hyperarousal. Because hypervigilance is theoretically related to (Ehlers \& Clark, 2000) and empirically predictive of (Norman, Stein, \& Davidson, 2007) significant functional impairment, a self-report measure of hypervigilance that captures a wider range of hypervigilant cognitions and behaviors may be particularly useful to researchers. To our knowledge, there are just two self-report measures of posttraumatic hypervigilance: the 11-item Hypervigilance Questionnaire (Kimble, Fleming, \& Bennion, 2009), and the 52-item Hypervigilance Questionnaire (HVQ; Knight, 1993; Knight \& Herwitz, 2010)_-both of which were developed within the context of research and clinical work with military veterans. Thus, there remains a need for a questionnaire for use in nonmilitary samples.

\section{The Current Study}

In the current study, we present the construction and psychometric properties of a new, abridged version of Knight's HVQthe Brief Hypervigilance Scale (BHS; see the Appendix)-intended for use in research with college samples (Aim 1). Second, we present preliminary findings on the associations between BHS hypervigilance scores, history of traumas involving varying degrees of betrayal, and dissociation (Aim 2). We anticipated that hypervigilance would relate to a history of both low betrayal trauma (e.g., natural disaster, automobile accident) and betrayal trauma (both of which can be frightening), whereas dissociation would relate to history of betrayal trauma but not history of low betrayal trauma.

\section{Method}

\section{Participants}

Participants were 510 undergraduates from a large public university in the Pacific Northwest. Participants were enrolled in one

\footnotetext{
${ }^{1}$ And presumably, traumas high on both dimensions should lead to both posttraumatic responses.

${ }^{2}$ A PsycNET search (11/14/2013) of publications with 'hypervigilance' in the title yielded 41 peer-reviewed journal articles and seven dissertation abstracts. Two of those dissertations and 18 articles came out of the pain literature, which uses the term hypervigilance to refer to the related but distinct phenomenon of enhanced pain sensitivity/reactivity - often seen in individuals with chronic pain conditions such as Fibromyalgia and Irritable Bowel Syndrome (e.g. Rollman, 2009). After removing these 20 publications, just over two dozen titles remained. By comparison, an analogous search with 'dissociation' yielded 2,975 peer-reviewed journal articles, 722 books, and 253 dissertations.
} 
of several introductory-level psychology or linguistics course, and completed the study for course credit. Twenty-one participants were dropped from analyses because of data missingness. Our final sample of 489 students (mean age $=19.92, S D=3.31$ ) was representative of the University's human subjects pool in gender (62.6\% female) and ethnicity (69.9\% Caucasian; $13.9 \%$ Asian American, 2.2\% African American; 1.6\% Pacific Islander; 0.8\% Native American; $10.8 \%$ other).

\section{Procedures}

Participants gave consent, completed a short demographic questionnaire and four self-report measures (in the order shown below), and were debriefed about the purposes of the study via Qualtrics online survey software. The study protocol was approved by the host University's Committee for the Protection of Human Subjects.

Hypervigilance. The 52-item HVQ (version 2.01; Knight, 1993; Knight \& Herwitz, 2010) was developed to capture many of the hypervigilant behaviors commonly endorsed by veterans with PTSD, and contains items about discomfort in crowds, feeling unsafe at home, planning for and preparing to escape danger, reactive and static vigilance, scanning, and fatigue from scanning. The HVQ uses a 5-point scale from 0 (not at all like me/never true) to 4 (very much like melalways true). In this sample, Cronbach's alpha for the HVQ was very good $(\alpha=.84)$, though the average interitem correlation was low, $r=.10$ (range $=-.46$ to .68 ).

PTSD symptoms. The PCL-C contains 17 items measuring PTSD symptoms (Blanchard et al., 1996) using a 5-point scale from 1 (not at all) to 5 (extremely), yielding total scores between 17 and 85 . A sample item is "in the past month, how much were you been bothered by: repeated, disturbing, and unwanted memories of the stressful experience?" In this sample, PCL-C reliability was excellent $(\alpha=.93)$. Based on National Center for PTSD scoring guidelines, 100 participants $(20.44 \%)$ met Diagnostic and Statistical Manual of Mental Disorders, fourth edition, text revision (DSM-IV-TR; American Psychiatric Association, 2000) criteria for PTSD.

Dissociation. The six-item dissociation subscale of the Trauma Symptom Checklist (TSC; Elliott \& Briere, 1992) assesses dissociative symptoms on a 4-point scale ranging from 0 (never) to 3 (often). Instructions ask participants, "How often have you experienced each of the following in the last month?" A sample item is "Spacing out (going away in your mind)." In this sample, reliability for the subscale was very good $(\alpha=.81)$.

Trauma history. The Brief Betrayal Trauma Survey (BBTS; Goldberg \& Freyd, 2006) assesses participants' experience of 14 different traumas (before and after 18 years of age) using a three-point scale: 0 (never), 1 (one or two times), and 2 (more than that). Six sum-scored subscales were computed for childhood and adulthood histories of low betrayal traumas (LBT; e.g., a natural disaster), medium betrayal traumas (MBT; e.g., physical abuse by someone not close to the respondent), and high betrayal traumas (HBT; e.g., sexual abuse by someone very close to the respondent). Using these scores, we computed composite variables representing total childhood betrayal trauma (the sum of childhood HBT and MBT), total adult betrayal trauma (the sum of adulthood HBT and MBT), total lifetime betrayal trauma (the sum of the previous two composites), and total lifetime low betrayal trauma (the sum of
LBT items in childhood and adulthood). The BBTS has been found have high test-retest reliability over a period of three years (Goldberg, \& Freyd, 2006).

\section{BHS Scale Construction}

The BHS scale was constructed with a psychometric evaluation of the hypervigilance items developed by Knight (1993). ${ }^{3}$ Using psychological scale construction guidelines outlined by Simms (2008), we evaluated Knight's 52-item pool with a non-treatmentseeking sample, with the goal of identifying a small subset of items that were appropriate for an undergraduate sample. The theoretical core of the construct we predicted would manifest across populations was "being super alert or watchful, on guard"- the hypervigilance item (\#16) of the PCL-C.

Using roughly half of the dataset $(n=228),{ }^{4}$ we conducted an internal consistency analysis with the PCL-C hypervigilance item and all 52 HVQ items to identify HVQ items that correlated with the hypervigilance item at a strength of $r=.30$ or higher. Six items met this criterion (see Table 1). To validate these six items, we repeated the analysis with the second half of the dataset $(n=254)$. In this subsample, there were also six items that met this criterion-five of which were the same and one was novel. For our final scale, we retained those five items (i.e., "BHS items") that met our retention criterion in both halves of the dataset.

\section{Results}

Descriptive statistics for the sample can be found in Table 2 and their intercorrelations in Table 3. Prevalence of trauma history within the sample was similar to other studies using college samples (e.g., Gobin, 2012). For all three levels of betrayal (low, medium, and high), approximately two-fifths of the current sample reported experiencing at least one trauma before the age of 18 (37.9\% of the sample for high; $42.6 \%$ for medium; and $40.4 \%$ for low betrayal traumas). Slightly fewer reported experiencing at least one trauma after the age of 18 (28.3\% of the sample for high, $32.3 \%$ for medium, and $29.7 \%$ for low betrayal traumas), which is unsurprising given the sample's young age.

\section{BHS Psychometric Properties and Descriptives}

All five BHS items loaded onto a single factor explaining $57.32 \%$ of the variance, and scale reliability was very good $(\alpha=$ .81 ; average interitem correlation $=0.46$ [range $=0.35-0.59])$. In a linear regression, BHS total score accounted for $69.0 \%$ of the

\footnotetext{
${ }^{3}$ An initial Principle Components Factor analysis of the 52 HVQ items revealed four factors explaining $20.44 \%, 8.44 \%, 5.35 \%$, and $3.88 \%$ of the variance in HVQ, respectively. An examination of factor loadings revealed that while many items (especially those on scanning and escape-planning) loaded onto Component 1 , fewer loaded onto the others. Items loading onto the other components seemed to more closely represent self-protection (i.e. having a nightly outside security-checking routine, needing to carry/sleep with a weapon to feel safe); adventurousness/extraversion (i.e. enjoying unpredictability, social gatherings, and public outings); and introversion (i.e. preferring a quiet, empty home and being home alone), respectively. We interpreted these results as indicating that HV was unidimensional within our non-veteran college sample.

${ }^{4}$ Sample halves did not differ from one another on any focal or demographic variable.
} 
Table 1

HVQ Items Meeting Retention Criterion for Each Half of the Sample

\begin{tabular}{|c|c|c|c|}
\hline \multirow[b]{2}{*}{ Item No. } & \multirow[b]{2}{*}{ HVQ item } & \multicolumn{2}{|c|}{$r$ value } \\
\hline & & $\begin{array}{l}\text { Sample } 1 \\
(n=228)\end{array}$ & $\begin{array}{l}\text { Sample } 2 \\
(n=254)\end{array}$ \\
\hline 17 & Sudden noises make me very alert, watchful, and preoccupied with things going on around me & .27 & .31 \\
\hline $22^{\mathrm{a}}$ & As soon as I wake up and for the rest of the day, I am watching for signs of trouble & .35 & .34 \\
\hline $29^{\mathrm{a}}$ & $\begin{array}{l}\text { When I am outside, I think ahead about what I would do (or where I would go) if someone would try to } \\
\text { surprise or harm me }\end{array}$ & .42 & .34 \\
\hline $32^{\mathrm{a}}$ & I notice that when I am in public or new places, I need to scan the crowd or surroundings & .31 & .38 \\
\hline $36^{\mathrm{a}}$ & When I am in public, I feel overwhelmed because I cannot keep track of everything going on around me & .30 & .30 \\
\hline 44 & When I enter a new place, I make sure that I know where the exits and stairs are located & .30 & .23 \\
\hline $49^{\mathrm{a}}$ & I feel that if I don't stay alert and watchful, something bad will happen & .31 & .50 \\
\hline
\end{tabular}

Note. Bolded $r$ values are $\geq .30$

${ }^{\mathrm{a}}$ Item retained for final BHS.

variance in HVQ total score. BHS scores did not significantly differ between males and females, $t(430.62)=1.56, p=.12$, or Caucasian and non-Caucasian individuals, $t(474)=-0.96, p=$ .34. As Kimble and colleagues (2013) have found with their 11-item Hypervigilance Questionnaire, we detected a weak correlation between BHS scores and age, $r=.10, p=.03$.

In evaluating the convergent validity of the BHS, we found that BHS scores were moderately related to total past-year PTSD symptoms, $r=.50, p<.001$. Examination of the separate PTSD symptom cluster scores revealed that BHS had comparable correlations with each $(r=.44$ with reexperiencing, $r=.41$ with avoidance/numbing, and $r=.44$ with hyperarousal, all $p<.001$ ). In linear regressions, BHS scores explained $24 \%$ of the variance in past-year PCL-C total scores, and $22 \%$ of the variance in past-year hyperarousal cluster scores (the cluster that includes hypervigilance). BHS scores were also positively related to all six forms of trauma experience assessed by the BBTS, with $r$ values ranging from 0.16 (for low BT before 18) to 0.30 (for BT after 18).

\section{BHS Psychometric Properties and Descriptives for Individuals With PTSD}

We also examined the psychometric properties of the BHS for the subset of participants $(n=100)$ who indicated clinically significant levels of posttraumatic stress on the PCL-C. Within this subsample, BHS reliability remained high $(\alpha=.77$; average interitem correlation $=0.40$ [range $=0.29-0.54]$ ). In a linear regression, BHS scores continued to account for a large portion $(67.5 \%)$ of the variance in HVQ scores. As we had found with the entire sample, BHS scores did not differ between males $(n=29)$ and females $(n=66), t(93)=0.13, p=.89$, or Caucasian $(n=$ 64) and non-Caucasian individuals $(n=32), t(94)=-0.23, p=$ .82 . Within this subsample, age and BHS were no longer correlated, $r=.04, p=.68, n s$.

As was the case in the whole sample, BHS scores were associated with past-year PCL-C total score, $r=.29, p=.004$, and had comparable correlations with each symptom cluster $(r=.23, p=$

Table 2

Descriptive Statistics by PTSD Diagnostic Status

\begin{tabular}{|c|c|c|c|c|c|c|c|}
\hline \multirow[b]{2}{*}{ Variable } & \multicolumn{2}{|c|}{$\begin{array}{l}\text { No PTSD } \\
(n=389)\end{array}$} & \multicolumn{2}{|c|}{$\begin{array}{c}\text { PTSD } \\
(n=100)\end{array}$} & \multirow[b]{2}{*}{$t$} & \multicolumn{2}{|c|}{$\begin{array}{c}\text { Total } \\
(n=489)\end{array}$} \\
\hline & $M$ & $S D$ & $M$ & $S D$ & & $M$ & $S D$ \\
\hline Age & 19.88 & 3.45 & 20.07 & 2.72 & 0.59 & 19.92 & 3.31 \\
\hline \multicolumn{8}{|l|}{ Hypervigilance } \\
\hline BHS (5 items) & 3.72 & 3.21 & 7.79 & 4.44 & $8.61^{* *}$ & 4.54 & 3.85 \\
\hline HVQ (52 items) & 75.94 & 17.09 & 94.78 & 18.99 & $9.03^{* *}$ & 79.78 & 19.04 \\
\hline Total PTSD symptoms & 28.31 & 8.33 & 52.49 & 9.35 & $23.57^{* *}$ & 33.28 & 12.99 \\
\hline Dissociation & 4.19 & 3.54 & 9.28 & 4.43 & $10.65^{* *}$ & 5.24 & 4.25 \\
\hline \multicolumn{8}{|l|}{ BT history } \\
\hline \multicolumn{8}{|l|}{ Childhood } \\
\hline High & 0.59 & 1.16 & 2.11 & 1.85 & $7.83^{* *}$ & 0.90 & 1.47 \\
\hline Medium & 0.71 & 1.33 & 2.32 & 2.27 & $6.80^{* *}$ & 1.03 & 1.69 \\
\hline Low & 0.61 & 0.98 & 1.35 & 1.69 & $4.20^{* * *}$ & 0.76 & 1.19 \\
\hline \multicolumn{8}{|l|}{ Adulthood } \\
\hline High & 0.34 & 0.80 & 1.53 & 1.63 & $7.08^{* *}$ & 0.59 & 1.14 \\
\hline Medium & 0.47 & 0.98 & 1.88 & 2.26 & $6.09^{* *}$ & 0.75 & 1.44 \\
\hline Low & 0.43 & 0.88 & 1.13 & 1.64 & $4.12^{* *}$ & 0.58 & 1.11 \\
\hline
\end{tabular}

Note. $\quad \mathrm{BT}=$ Betrayal trauma

*** $p<.001$. 
Table 3

Variable Intercorrelations

\begin{tabular}{|c|c|c|c|c|c|c|c|c|c|c|}
\hline Variable & 1 & 2 & 3 & 4 & 5 & 6 & 7 & 8 & 9 & 10 \\
\hline 1. Age & - & & & & & & & & & \\
\hline 2. BHS & $.10^{*}$ & - & & & & & & & & \\
\hline 3. HVQ & $.13^{*}$ & $.83^{* *}$ & - & & & & & & & \\
\hline 4. PCL-C & .05 & $.50^{* *}$ & $.49^{\text {** }}$ & - & & & & & & \\
\hline 5. Dissociation & -.05 & $.38^{* * *}$ & $.36^{* * *}$ & $.70^{* * *}$ & - & & & & & \\
\hline 6. Child High BT & $.11^{*}$ & $.28^{* * *}$ & $.24^{* * *}$ & $.53^{\text {*** }}$ & $.43^{\text {*** }}$ & - & & & & \\
\hline 7. Child Med BT & $.11^{*}$ & $.26^{* *}$ & $.23^{* *}$ & $.48^{* * *}$ & $.43^{* * *}$ & $.65^{* * *}$ & - & & & \\
\hline 8. Child Low BT & .02 & $.16^{* * *}$ & $.16^{* * *}$ & $.34^{* * *}$ & $.29^{* * *}$ & $.31^{* * *}$ & $.57^{* * *}$ & - & & \\
\hline 9. Adult High BT & $.17^{* * *}$ & $.30^{* *}$ & $.27^{* * *}$ & $.49^{* * *}$ & $.35^{\text {*** }}$ & $.69^{* * *}$ & $.55^{\text {** }}$ & $.27^{* * *}$ & - & \\
\hline 10. Adult Med BT & .08 & $.27^{* * *}$ & $.24^{* * *}$ & $.46^{* * *}$ & $.38^{* * *}$ & $.49^{* *}$ & $.75^{* * *}$ & $.50^{* *}$ & $.65^{* *}$ & - \\
\hline 11. Adult Low BT & .05 & $.19^{* * *}$ & $.19^{* * *}$ & $.29^{* * *}$ & $.22^{* * *}$ & $.20^{\text {*** }}$ & $.44^{* * *}$ & $.72^{* *}$ & $.33^{* *}$ & $.59^{* * *}$ \\
\hline
\end{tabular}

Note. $\quad \mathrm{BT}=$ Betrayal trauma.

${ }^{*} p<.05$. ** $p<.001$.

.03 with reexperiencing; $r=.34, p=.001$ with avoidance/ numbing; and $r=.25, p=.01$ with hyperarousal). A binary logistic regression, $\chi^{2}(1)=79.86, p<.001$, revealed that BHS scores predicted $24.4 \%$ of the variation in PTSD diagnostic status, and correctly classified $95.3 \%$ of individuals without, and $28.1 \%$ of those with PTSD. BHS score remained a significant predictor of PTSD diagnostic status $(B=.18$, Wald [1] $=16.05, p<.001)$ controlling for past year reexperiencing and avoidance/numbing cluster scores, $\chi^{2}(3)=233.20, p<.001$. Likely a result of the constricted range within this symptomatic subsample, BHS scores were no longer correlated with any trauma experience, though the correlation between BHS scores and adulthood high BT was trending, $r=.20, p=.07$.

\section{Trauma, Hypervigilance, and Dissociation}

In line with what Kimble and colleagues (2013) found with their hypervigilance scale, we detected a moderate positive zero-order correlation between hypervigilance and dissociation, $r=.38, p<$ .001. Partial correlations tested the independent associations among hypervigilance, dissociation, and lifetime experiences of traumas with elevated and low levels of betrayal (BT and LBT, respectively). The correlation between hypervigilance and BTcontrolling for dissociation and LBT-was significant, $r=.15$, $p=.002$, as was the correlation between dissociation and BTcontrolling for hypervigilance and LBT, $r=.35, p<.001$. The same two partial correlations were nonsignificant when LBT and BT were reversed $(r=.00, p=.96, n s$ for $L B T$ and hypervigilance controlling for BT and dissociation; $r=.01, p=.77, n s$ for $L B T$ and dissociation controlling for BT and hypervigilance).

Because there is evidence from the developmental trauma literature indicating that relational traumas occurring during childhood may lead to qualitatively different outcomes than those occurring in adulthood (Briere \& Elliott, 2003; Cicchetti \& Olsen, 1990; Pearlman, 1997; van Der Kolk, 1996), we reexamined the two significant correlations above parceling apart childhood and adulthood experiences of BT. We found the association between hypervigilance and childhood BT - controlling for dissociation, lifetime LBT, and adulthood BT-was not significant, $r=.01, p=$ $.91, n s$, though the association between dissociation and childhood BT-controlling for hypervigilance, lifetime LBT, and adulthood BT-was, $r=.21, p<.001$. The reverse pattern of significance was found when child and adult BT were inverted $(r=.12, p=$ .011 for adulthood BT and hypervigilance controlling for dissociation, lifetime LBT, and childhood BT; and $r=.08, p=.10, n s$ for adulthood BT and dissociation controlling for hypervigilance, lifetime LBT, and childhood BT).

Finally, because BTT holds that the betrayal inherent to a trauma is highest-and the adaptiveness of dissociation strongest-when a person's relationship to their perpetrator is closer and more necessary, we repeated the same analyses for this second pair of significant correlations, parceling apart BTs with high (HBT) and medium (MBT) levels of betrayal. We found that although the association between hypervigilance and adulthood $H B T$ - controlling for dissociation, lifetime LBT, childhood BT, and adulthood MBT-was significant, $r=.13, p=.011$, the association between hypervigilance and adulthood MBT - controlling for dissociation, lifetime LBT, childhood BT, and adulthood HBT_-was not, $r=.05, p=.30, n s$. In contrast, we found that the association between dissociation and childhood HBT (controlling for hypervigilance, lifetime LBT, adulthood BT, and childhood MBT) and the association between dissociation and childhood $M B T$ (controlling for hypervigilance, lifetime LBT, adulthood BT, and childhood HBT) were both significant, $r=.15, p=.003$ and $r=.11, p=.024$, respectively.

\section{Discussion}

In this report, we have described the development of the 5-item BHS and provided initial psychometric and descriptive results for (a) a college sample, and (b) the subset of undergraduates who met diagnostic threshold for PTSD on the PCL-C. Results suggest that the BHS is a reliable measure for both populations. Interestingly, although we expected BHS scores to be more closely related to hyperarousal cluster scores relative to the other clusters, they related to all clusters equally. This finding is consistent with other research demonstrating moderate associations among clusters (e.g., Guerra \& Calhoun, 2013). Nevertheless, that BHS remained a significant predictor of diagnostic status while controlling for reexperiencing and avoidance/numbing symptoms suggests that the BHS explains unique variance in PTSD diagnostic status not accounted for by symptoms from other clusters.

Within the PTSD subsample, no significant correlations emerged between hypervigilance and trauma history, indicating 
that for traumatized individuals meeting criteria for PTSD, the association between trauma history and hypervigilance is not dose dependent. In other studies, researchers have found that number of unique types of trauma experienced (i.e., sexual, physical, and emotional abuse) is more predictive of negative outcomes than number of traumatic events (e.g., Arata, Langhinrichsen-Rohling, Bowers, \& O'Farrill-Swails, 2005; Finkelhor, Ormrod, \& Turner, 2007). These relationships deserve further exploration in future research.

\section{Hypervigilance, Betrayal Trauma, and Dissociation}

Results of the current study suggest that BHS-measured hypervigilance is most closely associated with adulthood betrayal trauma (BT) relative to other types of trauma (i.e., childhood BT; lifetime low BT). Although BTT had originally emphasized the link between betrayal and dissociation-not betrayal and hypervigilance-these findings are nevertheless consistent with several studies that have found that betrayal predicts anxiety (e.g., Klest \& Freyd, 2007; Goldsmith, Chesney, Heath, \& Barlow, 2013)—a cognitive, affective, and physiological state characterized by hypervigilance toward potential threats, not an unawareness of them. Because we found that the relation between adulthood BT and hypervigilance remained significant controlling for dissociation, it appears that hypervigilance and dissociation are independent correlates of BT, and that the relationship between hypervigilance and BT cannot be better explained by overlapping variance between hypervigilance and dissociation.

When we examined the relationships between hypervigilance and BT experiences during childhood and adulthood separately, we found that whereas dissociation related to childhood and not adulthood BT hypervigilance related to adulthood BT but not childhood BT. This interesting set of findings suggests that BT occurring in adulthood (or perhaps just in the more recent past) is most important in predicting hypervigilance, whereas BT occurring earlier in development is most important in predicting later dissociation. This finding is consistent with prior research within the developmental trauma literature indicating that relational traumas occurring during childhood lead to qualitatively different outcomes than those occurring in adulthood (e.g., Briere \& Elliott, 2003; Schimmenti, 2012; van der Kolk, 2005).

Our most unexpected finding was that hypervigilance was related to adulthood experiences of high BT, but not adulthood medium BT. Because items in the BHS assessing hypervigilance ask questions such as, "When I am outside, I think ahead about what I would do (or where I would go) if someone would try to surprise or harm me" and "When I am in public, I feel overwhelmed because I cannot keep track of everything going on around me"-items that connote a sense of 'stranger danger' - we might infer that young adults who have experienced high BT in adulthood might be demonstrating displaced posttraumatic hypervigilance. In other words, even though they were traumatized by someone with whom they were very close, they endorse hypervigilance toward environmental threats outside these close relationships. On the other hand, interpersonal traumatization by acquaintances or unfamiliar others was not associated with the type of stranger-danger vigilance we might expect. These unexpected findings may help explain how dissociation and hypervigilance can overlap within individuals: if an individual exhibits hypervigi- lance in the presence of strangers while maintaining unawareness of the ongoing danger presented by a close relationship, this represents a "dissociation" of important social information.

\section{Limitations and Future Directions}

The primary limitations of this study entail its exclusive reliance on self-report measures and limited sample demographics. Subsequent studies should examine the BHS in conjunction with behavioral measures of hypervigilance, and civilian samples with a wider range of ages, socioeconomic statuses, and ethnicities. Subsequent analyses with clinical samples should use diagnostic interviews to confirm PCL-C diagnoses. In addition, future research should further establish the BHS's discriminant validity by examining it in conjunction with self-report instruments of other symptoms (e.g., depression) and personality features (e.g., neuroticism). Pending further validation, the BHS holds substantial promise as an expedient research tool to fill an important gap in the currently available catalogue of assessments. Indeed, we have reported preliminary findings that speak to the measure's potential to test and refine hypotheses proposed by BTT.

\section{References}

American Psychiatric Association. (2000). Diagnostic and statistical manual of mental disorders, fourth edition, text revision. Washington, DC: Author.

Amstadter, A. B., Nugent, N. R., \& Koenen, K. C. (2009). Genetics of PTSD: Fear conditioning as a model for future research. Psychiatric Annals, 39, 358-367. http://dx.doi.org/10.3928/00485713-20090526-01

Arata, C. M., Langhinrichsen-Rohling, J., Bowers, D., \& O'Farrill-Swails, L. (2005). Single versus multi-type maltreatment: An examination of the long-term effects of child abuse. Journal of Aggression, Maltreatment \& Trauma, 11, 29-52. http://dx.doi.org/10.1300/J146v11n04_02

Arntz, A., Appels, C., \& Sieswerda, S. (2000). Hypervigilance in borderline disorder: A test with the emotional Stroop paradigm. Journal of Personality Disorders, 14, 366-373. http://dx.doi.org/10.1521/pedi .2000.14.4.366

Bernstein, E. M., \& Putnam, F. W. (1986). Development, reliability, and validity of a dissociation scale. Journal of Nervous and Mental Disease, 174, 727-735. http://dx.doi.org/10.1097/00005053-198612000-00004

Blanchard, E. B., Jones-Alexander, J., Buckley, T. C., \& Forneris, C. A. (1996). Psychometric properties of the PTSD Checklist (PCL). Behaviour Research and Therapy, 34, 669-673. http://dx.doi.org/10.1016/ 0005-7967(96)00033-2

Brett, E. A. (1996). The classification of posttraumatic stress disorder. In B. A. van der Kolk, A. C. McFarlane, \& L. Weisaeth (Eds.), Traumatic stress: The effects of overwhelming experience on mind, body, and society (pp. 117-128). New York, NY: Guilford Press.

Briere, J. (2006). Dissociative symptoms and trauma exposure: Specificity, affect dysregulation, and posttraumatic stress. Journal of Nervous and Mental Disease, 194, 78-82. http://dx.doi.org/10.1097/01.nmd .0000198139 .47371 .54

Briere, J., \& Elliott, D. M. (2003). Prevalence and psychological sequelae of self-reported childhood physical and sexual abuse in a general population sample of men and women. Child Abuse \& Neglect, 27, 12051222. http://dx.doi.org/10.1016/j.chiabu.2003.09.008

Cicchetti, D., \& Olsen, K. (1990). The developmental psychopathology of child maltreatment. In M. Lewis \& S. Miller (Eds.), Handbook of developmental psychopathology (pp. 261-279). New York, NY: Plenum Press. http://dx.doi.org/10.1007/978-1-4615-7142-1_21

Cisler, J. M., Wolitzky-Taylor, K. B., Adams, T. G., Jr., Babson, K. A., Badour, C. L., \& Willems, J. L. (2011). The emotional Stroop task and 
posttraumatic stress disorder: A meta-analysis. Clinical Psychology Review, 31, 817-828. http://dx.doi.org/10.1016/j.cpr.2011.03.007

Dalgleish, T., Moradi, A. R., Taghavi, M. R., Neshat-Doost, H. T., \& Yule, W. (2001). An experimental investigation of hypervigilance for threat in children and adolescents with post-traumatic stress disorder. Psychological Medicine, 31, 541-547. http://dx.doi.org/10.1017/S0033291701003567

De Cort, K., Hermans, D., Spruyt, A., Griez, E., \& Schruers, K. (2008). A specific attentional bias in panic disorder? Depression and Anxiety, 25, 951-955. http://dx.doi.org/10.1002/da.20376

DePrince, A. P., \& Freyd, J. J. (2002). The harm of trauma: Pathological fear, shattered assumptions, or betrayal? In J. Kauffman (Ed.), Loss of the assumptive world: A theory of traumatic loss (pp. 71-82). New York, NY: Brunner-Routledge.

DePrince, A. P., \& Freyd, J. J. (2007). Trauma-induced dissociation. In M. J. Friedman, T. M. Keane, \& P. A. Resick (Eds.), Handbook of PTSD: Science \& practice (pp. 135-150). New York, NY: Guilford Press.

Ehlers, A., \& Clark, D. M. (2000). A cognitive model of posttraumatic stress disorder. Behaviour Research and Therapy, 38, 319-345. http:// dx.doi.org/10.1016/S0005-7967(99)00123-0

Elliott, D. M., \& Briere, J. (1992). Sexual abuse trauma among professional women: Validating the Trauma Symptom Checklist-40 (TSC-40). Child Abuse \& Neglect, 16, 391-398. http://dx.doi.org/10.1016/0145-2134 (92) $90048-\mathrm{V}$

Fanselow, M. S., \& Ponnusamy, R. (2008). The use of conditioning tasks to model fear and anxiety. In R. J. Blanchard, D. C. Blanchard, G. Griebel, \& D. Nutt (Eds.), Handbook of anxiety and fear (pp. 29-48). San Diego, CA: Elsevier Academic. http://dx.doi.org/10.1016/S15697339(07)00003-3

Finkelhor, D., Ormrod, R. K., \& Turner, H. A. (2007). Poly-victimization: A neglected component in child victimization. Child Abuse \& Neglect, 31, 7-26. http://dx.doi.org/10.1016/j.chiabu.2006.06.008

Frankel, K. A., Boetsch, E. A., \& Harmon, R. J. (2000). Elevated picture completion scores: A possible indicator of hypervigilance in maltreated preschoolers. Child Abuse \& Neglect, 24, 63-70. http://dx.doi.org/ 10.1016/S0145-2134(99)00110-6

Freeman, D., Garety, P. A., \& Phillips, M. L. (2000). An examination of hypervigilance for external threat in individuals with generalized anxiety disorder and individuals with persecutory delusions using visual scan paths. The Quarterly Journal of Experimental Psychology A: Human Experimental Psychology, 53, 549-567. http://dx.doi.org/10.1080/ 713755896

Freyd, J. J. (1994). Betrayal trauma: Traumatic amnesia as an adaptive response to childhood abuse. Ethics \& Behavior, 4, 307-329. http://dx .doi.org/10.1207/s15327019eb0404_1

Freyd, J. J. (1996). Betrayal trauma: The logic of forgetting childhood abuse. Cambridge, MA: Harvard University Press.

Gobin, R. L. (2012). Partner preferences among survivors of betrayal trauma. Journal of Trauma \& Dissociation, 13, 152-174. http://dx.doi .org/10.1080/15299732.2012.642752

Goldberg, L. R., \& Freyd, J. J. (2006). Self-reports of potentially traumatic experiences in an adult community sample: Gender differences and test-retest stabilities of the items in a brief betrayal-trauma survey. Journal of Trauma \& Dissociation, 7, 39-63. http://dx.doi.org/10.1300/ J229v07n03_04

Goldsmith, R. E., Chesney, S. A., Heath, N. M., \& Barlow, M. R. (2013). Emotion regulation difficulties mediate associations between betrayal trauma and symptoms of posttraumatic stress, depression, and anxiety. Journal of Traumatic Stress, 26, 376-384. http://dx.doi.org/10.1002/jts .21819

Goldsmith, R. E., Freyd, J. J., \& DePrince, A. P. (2012). Betrayal trauma: Associations with psychological and physical symptoms in young adults. Journal of Interpersonal Violence, 27, 547-567. http://dx.doi .org/10.1177/0886260511421672
Guerra, V. S., Calhoun, P. S., \& the Mid-Atlantic Mental Illness Research, Education and Clinical Center Workgroup. (2011). Examining the relation between posttraumatic stress disorder and suicidal ideation in an OEF/OIF veteran sample. Journal of Anxiety Disorders, 25, 12-18. http://dx.doi.org/10.1016/j.janxdis.2010.06.025

Hailey, B. (2014, April). Betrayal trauma, risk detection, and substance use. Paper presented at meeting of the Western Psychological Association, Portland, OR, April 24-27, 2014.

Huijding, J., Mayer, B., Koster, E. H., \& Muris, P. (2011). To look or not to look: An eye movement study of hypervigilance during change detection in high and low spider fearful students. Emotion, 11, 666-674. http://dx.doi.org/10.1037/a0022996

Jones, E., \& Wessely, S. (2005). Shell shock to PTSD: Military psychiatry from 1900 to the Gulf War. London, UK: Psychology Press.

Kimble, M. O., Fleming, K., \& Bennion, K. (2009, November). The Hypervigilance Questionnaire: Assessment of hypervigilance in a trauma sample. Paper presented at the 25th Annual Meeting of the International Society for Traumatic Stress Studies. Atlanta, GA.

Kimble, M. O., Fleming, K., \& Bennion, K. A. (2013). Contributors to hypervigilance in a military and civilian sample. Journal of Interpersonal Violence, 28, 1672-1692. http://dx.doi.org/10.1177/0886260512468319

Klest, B. K., \& Freyd, J. J. (2007). Global ratings of essays about trauma: Development of the GREAT code, and correlations with physical and mental health outcomes. Journal of Psychological Trauma, 6, 1-20. http://dx.doi.org/10.1300/J513v06n01_01

Knight, J. A. (1993). The Hypervigilance Scale (Version 2.01). Unpublished measure. Boston, MA: Boston University School of Medicine.

Knight, J. A., \& Herwitz, C. (2010, August). Measuring clinical hypervigilance: Psychometric properties of the Hypervigilance Scale. Paper presented at the 118th Annual Convention of the American Psychological Association. San Diego, CA.

Martin, C. G., Cromer, L. D., Deprince, A. P., \& Freyd, J. J. (2013). The role of cumulative trauma, betrayal, and appraisals in understanding trauma symptomatology. Psychological Trauma: Theory, Research, Practice, and Policy, 5, 110-118. http://dx.doi.org/10.1037/a0025686

Milad, M. R., Orr, S. P., Lasko, N. B., Chang, Y., Rauch, S. L., \& Pitman, R. K. (2008). Presence and acquired origin of reduced recall for fear extinction in PTSD: Results of a twin study. Journal of Psychiatric Research, 42, 515-520. http://dx.doi.org/10.1016/j.jpsychires.2008.01 .017

Norman, S. B., Stein, M. B., \& Davidson, J. R. T. (2007). Profiling posttraumatic functional impairment. Journal of Nervous and Mental Disease, 195, 48-53. http://dx.doi.org/10.1097/01.nmd.0000252135 .25114 .02

Peach, N., Jovev, M., Foster, A., \& Jackson, H. J. (2012). Testing the Stroop effect in a nonclinical sample: Hypervigilance or difficulty to disengage? Journal of Experimental Psychopathology, 3, 496-510. http://dx.doi.org/10.5127/jep.017211

Pearlman, L. A. (1997). Trauma and the self: A theoretical and clinical perspective. Journal of Emotional Abuse, 1, 7-25. http://dx.doi.org/ 10.1300/J135v01n01_02

Peri, T., Ben-Shakhar, G., Orr, S. P., \& Shalev, A. Y. (2000). Psychophysiologic assessment of aversive conditioning in posttraumatic stress disorder. Biological Psychiatry, 47, 512-519. http://dx.doi.org/10.1016/ S0006-3223(99)00144-4

Rhodewalt, F., \& O'Keeffe, J. (1986). Type A behavior, field dependence, and hypervigilance: Toward increased Type A specificity. Motivation and Emotion, 10, 105-114. http://dx.doi.org/10.1007/BF00992250

Rollman, G. B. (2009). Perspectives on hypervigilance. Pain, 141, 183 184. http://dx.doi.org/10.1016/j.pain.2008.12.030

Schimmenti, A. (2012). Unveiling the hidden self: Developmental trauma and pathological shame. Psychodynamic Practice: Individuals, Groups 
and Organisations, 18, 195-211. http://dx.doi.org/10.1080/14753634 .2012 .664873

Simms, L. J. (2008). Classical and modern methods of psychological scale construction. Social and Personality Psychology Compass, 2, 414-433. http://dx.doi.org/10.1111/j.1751-9004.2007.00044.x

Sposari, J. A., \& Rapee, R. M. (2007). Attentional bias toward facial stimuli under conditions of social threat in socially phobic and nonclinical participants. Cognitive Therapy and Research, 31, 23-37. http://dx .doi.org/10.1007/s10608-006-9073-2

van der Kolk, B. A. (1996). The complexity of adaptation to trauma: Self-regulation, stimulus discrimination, and characterological devel- opment. In B. A. van der Kolk, A. C. McFarlane, \& L. Weisaeth (Eds.), Traumatic stress: The effects of overwhelming experience on mind, body, and society (pp. 182-213). New York, NY: Guilford Press.

van der Kolk, B. A. (2005). Developmental trauma disorder. Toward a rational diagnosis for children with complex trauma histories. Psychiatric Annals, 35, 401-410.

Wieser, M. J., Pauli, P., Weyers, P., Alpers, G. W., \& Mühlberger, A. (2009). Fear of negative evaluation and the hypervigilanceavoidance hypothesis: An eye-tracking study. Journal of Neural Transmission, 116, 717-723. http://dx.doi.org/10.1007/s00702-0080101-0

\section{Appendix}

\section{Brief Hypervigilance Scale}

Instructions: Please respond to each of the statements by placing an " $\mathrm{X}$ " in the answer column that best applies to you. There is no right or wrong response for each statement.

To help you decide your answer for each item, think back over the past month and then mark a column with your answer based either on "about how much the statement is true as it relates to you," or on "about how often the statement is true as it relates to you."

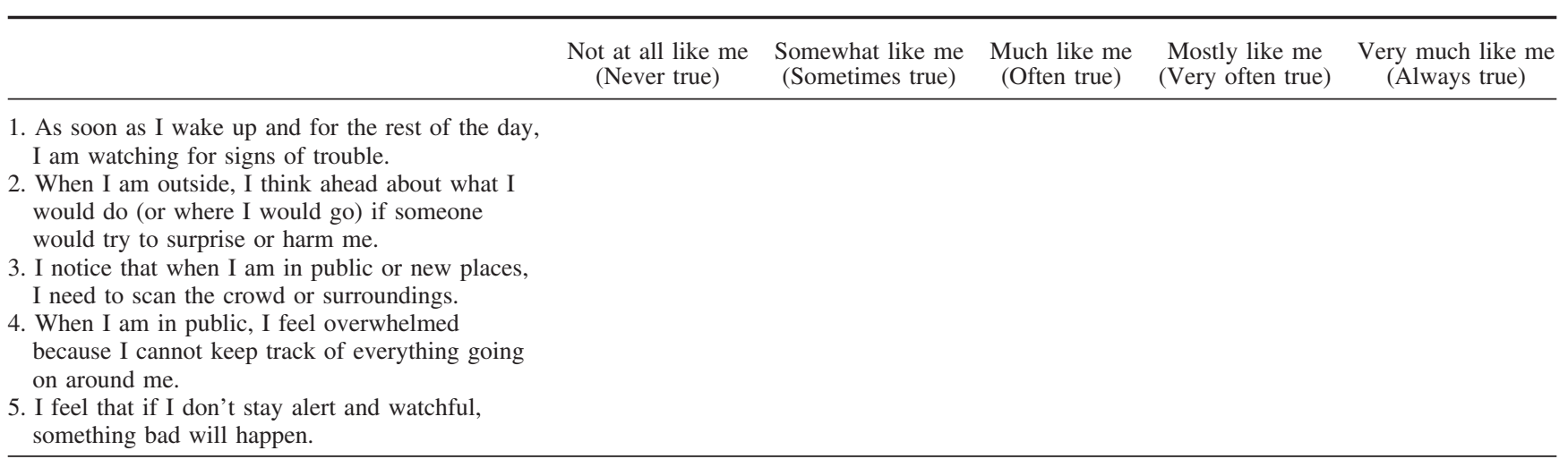

